\title{
Las redes de $\mathrm{I}+\mathrm{D}$ como estrategia de uso de las TIC en las universidades de América Latina
}

\author{
Jaim D. Royero
}

\section{Resumen}

El presente artículo tiene como propósito analizar el papel de las universidades como ejes de impulso en la configuración de redes avanzadas de I+D en América Latina, y en el uso y penetración de las TIC.

Dicho análisis parte de las consideraciones contextuales del papel de las TIC como consecuencia de la sociedad del conocimiento y la configuración internacional de los saberes, así como del entendimiento de las redes de I+D y su grado de vinculación social en el fortalecimiento de una sociedad tecnocultural.

Palabras clave

universidad, redes, investigación, TIC, América Latina, conocimiento

\section{SOCIEDAD DEL CONOCIMIENTO COMO MODELO DE TRANSFORMACIÓN DE LA EDUCACIÓN SUPERIOR}

La sociedad del conocimiento como expresión social ha tenido una consideración especial en torno al papel que juega en las transformaciones de los sistemas educativos en el mundo. ${ }^{1}$ Dentro de este cambio, los desafíos de la educación superior han sido mecanismos es-

\section{Abstract}

The aim of this paper is to analyze the role of universities as the driving forces in the establishment of advanced research and development $(\mathrm{R}+\mathrm{D})$ networks in Latin America, and their role in the penetration and ultimate use of information and communication technologies (ICT).

The analysis is based on the contextual characteristics of the role of ICT as shaped by the knowledge-based society and the international nature of knowledge, and on an appraisal of $\mathrm{R}+\mathrm{D}$ networks and their social impact in the role they play in strengthening a technocultural society.

\section{Key words}

university, networks, research, ICT, Latin America, knowledge.

\footnotetext{
${ }^{1}$ Véase Unesco (2005).

${ }^{2}$ Para destacar los desafíos de la educación superior ante esta realidad, véase BID (2003).
}

tratégicos para la expansión de los principios de la globalización en el auge del conocimiento y las nuevas tecnologías. $^{2}$

Dicha expansión se ha enmarcado dentro del contexto de las transformaciones esbozadas en la Declaración Mundial de Educación Superior, la Cumbre Mundial de la Sociedad de la Información y, muy especialmente, el Acuerdo General Sobre el Comercio de Servicios (GATS) auspiciado por la Organización Mundial del 
Comercio (OMC), que incluye la educación como objeto de intercambio.

Estos nuevos acuerdos, que abarcan el comercio de los servicios de educación, las innovaciones relacionadas con las técnicas de información y comunicación, y la importancia atribuida a la función del mercado, han generado cambios en los sistemas de educación en el globo (Unesco, 2004).

Dichos cambios han impactado determinantemente en las políticas de educación superior y en los discursos sobre ella, a partir de las siguientes implicaciones: ${ }^{3}$

1) La aparición de nuevos proveedores de educación, como las empresas multinacionales, universidades corporativas y compañías de medios de comunicación.

2) Las nuevas formas de proporcionar educación, entre ellas la educación a distancia, la educación virtual y el aprendizaje en contacto directo, como las empresas privadas.

3) La mayor diversificación de los diplomas y los títulos universitarios.

4) El aumento de la movilidad de los estudiantes, los programas, los proveedores de educación y los proyectos por fronteras nacionales.

5) El mayor hincapié en la enseñanza permanente, lo que a su vez aumenta la demanda de educación postsecundaria.

6) El incremento de la inversión privada en el suministro de la educación superior (Unesco, 2004, pág. 8).

No obstante, tales parámetros operan en contextos diferentes según la nación o región en particular, pero siempre están orientados a las necesidades sociales enmarcadas en la intención internacional del rol de los sistemas educativos en relación con las exigencias del modo de producción capitalista.

\section{USO Y PENETRACIÓN DE LAS TIC EN LA SOGIEDAD DEL CONOCIMIENTO: ¿LA BRECHA SE REDUCE?}

Como se afirmó en el apartado anterior, los cambios contextuales han afectado las características de la sociedad en relación con los canales de distribución y difusión de las tecnologías de la información y de la comunicación (TIC) en todas las regiones del mundo, y muy especialmente en América, y se ha configurado lo que se conoce como la brecha digital.

Esta brecha reconocida en la última reunión de los ministros de Relaciones Exteriores y jefes de delegación de los estados miembros de la Organización de los Estados Americanos (OEA), celebrada en el mes de junio de $2006,{ }^{4}$ pone al descubierto las múltiples necesidades de los países para reducirla en un marco de progreso sostenible.

En este sentido, la medición del grado de penetración y uso de las TIC en las sociedades modernas es llevada a cabo por organismos internacionales ${ }^{5}$ a fin de dar cuenta de los avances y limitaciones de las naciones en el cumplimiento de esta dinámica global.

Esta medición a partir de indicadores básicos, como el nivel de acceso a la energía eléctrica, el uso de la radio, la televisión y el teléfono (fijo o móvil), el número de ordenadores y el acceso a Internet por parte de los pobladores de una nación o región específica, constituye una fuente importante para destacar la caracterización de este proceso en la sociedad del conocimiento.

Dichos indicadores se aplican en tres niveles de medición básicos: el individual calcula la distribución proporcional per capita del uso de las TIC; el de servicio universal, la proporción de posicionamiento y empleo de las TIC en los hogares, y el comunitario, el alcance ge-

\footnotetext{
Para profundizar en este tema, veáse Unesco (2004)

* Véase OEA (2006).

La Unión Internacional de Telecomunicaciones, con sede en Suiza, ente adscrito a las Naciones Unidas, es uno de los organismos que facilita la medición y seguimiento de las TIC en el mundo.
} 
neral de la penetración de las TIC en una población determinada (UIT, 2003).

Estos requisitos están relacionados con una cultura tecnológica íntimamente vinculada al nivel de vida y a las condiciones socioeconómicas de la población que precisa su uso; por eso, en las sociedades en vías de desarrollo, estas relaciones son inversamente proporcionales en comparación con los países desarrollados.

Tal desproporción orienta una brecha inminente en el acceso a las TIC de algunos países sobre otros a partir de dos condiciones básicas: la posesión de recursos económicos para comprar dicha tecnología (ordenador, impresora, línea telefónica, proveedor de Internet, etc.) y la posesión consolidada de los conocimientos necesarios para el manejo de la TIC de forma efectiva, es decir, las condiciones para la formación tecnocultural (Domínguez, 2003).

En el último informe sobre el desarrollo mundial de las telecomunicaciones (UIT, 2006) se indica que, a medida que avanzan las tecnologías, sigue habiendo una brecha en cuanto al acceso a las aplicaciones más modernas de las TIC, tanto entre los países como entre los grupos sociales.

Afirma el documento que, en las regiones de baja penetración (como Asia y África), las tasas de crecimiento son superiores a la media. Si bien este fenómeno ha reducido en cierto grado la brecha digital, en 2004 Europa y América (con niveles de penetración del 41 y el $34 \%$, respectivamente) se hallaban por adelante de Asia $(14 \%)$ y registraban un nivel de penetración diez veces superior al de África (3\%). En el mismo orden indica que, a fines de 2004, alrededor del $28 \%$ de la población mundial estaba abonado a servicios de telefonía móvil, pero el $74 \%$ de esas personas habita en Asia o Europa.
Los servicios de telefonía móvil con tecnología 3G, según este informe, llegaron en 2005 a 150 millones de abonados en todo el mundo, lo que supone un aumento de casi el $60 \%$ en comparación con el año anterior. Sin embargo, en vez de ser una brecha regional, el mercado 3G separa a unos pocos países del resto del mundo. A finales de 2004, Estados Unidos, con 49,5 millones de abonados a servicios 3G, la República de Corea (27,5 millones) y Japón (25,7 millones) representaban conjuntamente las tres cuartas partes del total mundial.

Del mismo modo, las tasas de penetración más elevadas en Internet se registran en Europa y América, con casi una tercera parte de la población. En 2004, los abonados a los servicios de Internet de banda ancha representaban aproximadamente el 2,5\% de la población mundial y el $38 \%$ del total de abonados a la misma en todo el mundo. Y aunque en varios países más del $50 \%$ de la población utiliza Internet, el promedio en África sólo es del $2,6 \%$ y apenas un $0,1 \%$ de dichos usuarios se conecta con banda ancha (UIT, 2006).

Igualmente, las diferencias son claramente sustanciales en relación con la inversión de las TIC. En 2004 le correspondió a Asia la mayor proporción de inversiones en el área. En 2004, sólo en China las inversiones en telecomunicaciones ascendían a 27.000 millones de dólares norteamericanos, es decir, a casi el $15 \%$ del total mundial. A las regiones de Europa y América les correspondía el 33 y el 23\%, respectivamente, mientras que el porcentaje de África sólo era del $4 \%$.

En el caso de América Latina, las desigualdades entre los propios países en torno a la penetración y uso de las TIC son claras a partir de los resultados de los organismos regionales. ${ }^{6}$ Sin embargo, el aumento en la utilización de las TIC en el continente ha sido muy significativo. La penetración de Internet en la región ha crecido 
un $211 \%$ entre los años 2002 y 2005, pero sólo el 10,3\% tiene acceso a Internet y el 6,6\% dispone de un ordenador, comparados con el $67,9 \%$ y el $65,8 \%$ de Estados Unidos, respectivamente (BID, 2005).

En la región, se evidencian razones concretas que impiden la reducción de la brecha digital. Según el Banco Interamericano de Desarrollo (BID), estas razones se concentran en la escasa capacidad institucional para promover y articular políticas en el área, la limitada cobertura que ofrece la misma, los elevados costos del acceso, la falta de educación digital y la baja disponibilidad de contenido de interés regional y local.

A pesar de estas limitaciones estructurales que han impedido la reducción de la brecha digital y el uso masivo de las TIC en el continente, la diversificación de los centros de comunicación de información de carácter comercial y públicos ha sido un factor importante en la ampliación de la cobertura y penetración tecnológica. ${ }^{7}$ A ello hay que añadirle el desarrollo de políticas para la institucionalidad social y las TIC por parte de los gobiernos de América Latina. ${ }^{8}$

\section{REALIDADES Y PRIORIDADES DE LAS REDES DE I+D EN AMÉRICA LATINA COMO EXPRESIÓN DE LA SOCIEDAD DEL CONOCIMIENTO}

En el contexto más inmediato, las redes electrónicas de investigación y desarrollo $(\mathrm{I}+\mathrm{D})$ provienen de la evolución de la sociedad del conocimiento en el nacimiento de las redes en el sistema social o la llamada sociedad en red. «La sociedad en red [...] son las interacciones y flujos de símbolos generalizados de comunicación que sustituyen las determinaciones de uso personal y regional» (Domínguez, 2003, pág. 6).

Estas exigencias de la sociedad en red han influido enormemente en la dinámica de cooperación entre los centros de investigación en el ámbito mundial, y muy específicamente en las universidades como centros de conocimiento a partir del desarrollo de actividades de investigación, en lo que comúnmente se llaman redes de investigación.

Éstas son asociaciones de grupos de I+D para el desarrollo de actividades de investigación y avance tecnológico a partir de proyectos de estudio con el objeto de complementar capacidades y un adecuado reparto de actividades o tareas (Sebastián, 1999).

El auge de las redes de investigación se concentró en el llamado modelo de ramificación a partir de los años setenta, al definir que la comunidad científica tendió a organizarse en distintas formas institucionales (llámense redes, áreas, especialidades o disciplinas). Las nuevas áreas, junto con las redes de científicos, crearon a su vez redes sociales pequeñas y ajenas a las fronteras entre disciplinas científicas, pero que se intercambiaron a partir del propio interés del investigador en pertenecer a otra red u otra área (Olazarán et al., 1999).

En este sentido, a mediados de los años noventa, en América Latina, se apreciaron los primeros cambios en las políticas de ciencia y tecnología que fortalecieron el desarrollo de las redes de I+D y que fueron impulsadas por organismos internacionales como la CEPAL (Comisión Económica Para América Latina), el SELA (Sistema Económico Latinoamericano), el BID y la OEA. Dichos cambios se concentraron básicamente en la reorganización institucional de la gestión en los sistemas de ciencia y tecnología, así como en las modificaciones introducidas por las legislaciones que daban a los Estados una mayor responsabilidad en el fomento de las actividades de $\mathrm{I}+\mathrm{D}$, y la integración de las universidades y los centros de investigación en dicha estrategia (Sánchez, 2004).

A la par de esta realidad, se crearon iniciativas, como el programa CYTED (Programa Iberoamericano de Ciencia

\footnotetext{
${ }^{7}$ Para profundizar en el impacto de los centros de información en América Latina en la masificación de las TIC, consúltese Proenza et al. (2001).

Para un mejor entendimiento de esta relación, veáse CEPAL (2005)
} 
y Tecnología para el Desarrollo) y las nuevas relaciones de cooperación en el continente, como el Mercado Común de Ciencia y Tecnología (MERCOCYT) para el año 1993 y la Comisión Interamericana de Ciencia y Tecnología (COMCYT), patrocinada por la OEA, a partir de 1998.

Estos avances se evidenciaron claramente en el gasto de los países en el PIB (producto interior bruto) en relación con la $\mathrm{I}+\mathrm{D}$ al pasar de un $0,49 \%$ en 1990 a un $0,57 \%$ en 2003 , pero muy por debajo si lo comparamos con los países desarrollados, como Canadá $(1,91 \%)$ o Estados Unidos (2,58\%) para el mismo año (RICYT, 2005).

De igual modo, los niveles de cooperación en el ámbito iberoamericano tuvieron un auge significativo: desde 1990, sólo trece proyectos y una red temática configuraron dicha relación; en 2005, esta proporción creció sustancialmente con cuarenta y dos proyectos de investigación y cuarenta y cuatro redes temáticas.

Con el inicio de un nuevo modelo de desarrollo globalizador, dentro del contexto de la sociedad del conocimiento implantado a finales de los noventa y principios del presente siglo, más el auge de los sistemas de información y de comunicación avanzados como Internet, las relaciones de cooperación y desarrollo tecnológico constituyeron el catalizador necesario para la interconexión electrónica de dichas redes y su vinculación en el avance económico y social.

Las redes avanzadas de $\mathrm{I}+\mathrm{D}$ constituyen «un instrumento fundamental para impulsar a los países de la región hacia economías competitivas y basadas en el conocimiento». Entre otras cosas, «brinda acceso a los recursos científicos y tecnológicos de todo el mundo, y fortalece importantes actividades sociales y económicas» (OEA, 2005).

En América Latina, estas redes presentan grandes limitaciones y dificultades referidas a la poca conciencia en la vinculación estratégica entre el mantenimiento de redes avanzadas y la infraestructura de información para el desarrollo de políticas por parte de los países de la región, así como la existencia de marcos legales inapropiados para su creación y mantenimiento. De igual modo, en la creación de dichas redes se evidencia el poco uso de la banda ancha de gran capacidad a precios competitivos y la escasa disposición de recursos humanos para su consolidación en el continente (OEA, 2005).

Dentro de esta problemática, existen grandes diferencias entre países en torno a las capacidades y recursos, que ponen en duda una efectiva cooperación tecnológica interregional aunada a un inadecuado financiamiento para la investigación y educación en la creación de estructuras necesarias para el mantenimiento y avance de las mencionadas redes (OEA, 2005).

Brasil, México, Argentina y Chile representan casi la mitad de los gastos de I+D y del personal en ciencia y tecnología para América Latina, así como su aproximación a la media del continente en relación con los gastos a partir del PIB y de los gastos en I+D por habitante, en el mismo orden. Dicho bloque lidera, con casi un $90 \%$, los doctores titulados y supera el promedio estimado de investigadores por cada mil habitantes (RICYT, 2005).

Del mismo modo, este eje lidera la solicitud y otorgamiento de patentes del total registrado para América Latina y el Caribe, con un 82 y $87 \%$ respectivamente, al igual que los artículos científicos publicados en SCI al alcanzar 31.538 de los 35.229 para todo el continente sudamericano en el año 2003 (RICYT, 2005).

A pesar de la existencia de la Cooperación Latinoamericana de Redes Avanzadas (CLARA), ${ }^{9}$ en la región se han logrado aisladamente progresos en la creación de redes con tecnologías punta. La red CLARA ya forma parte del conjunto de redes mundiales conformadas por In-

\footnotetext{
${ }^{9}$ Los países que inicialmente han firmado los estatutos de CLARA hasta 2005 son: Argentina, Bolivia, Brasil, Chile, Costa Rica, Cuba, Ecuador, Honduras, México, Nicaragua, Paraguay, Panamá, Perú, El Salvador, Uruguay y Venezuela.
} 
ternet2, Canarie (Organización Canadiense para el Desarrollo de Internet Avanzado), GEANT (Red de Investigación Paneuropea), APAN (Consorcio Asiático-Pacífico de Redes Avanzadas) y Singaren (Corporación de Red de Investigación y Educación de Singapur).

Dicha cooperación, aunque no en bloque, ha ido desarrollándose con otros proyectos de envergadura, como es el caso de la red de Ameritas PATH, dirigida por la Universidad de Florida (FIU) en colaboración con Global Crossing (GC), que interconectará las redes de educación e investigación en Sudamérica y Centroamérica, el Caribe y México a las de investigación y educación dentro y fuera de Estados Unidos. Hasta 2005, sólo Brasil, México, Argentina y Chile participan en dicho proyecto.

Sin embargo, la mayoría de las redes nacionales de investigación y educación (NREN) están basadas en servicios comerciales de Internet a baja velocidad que le impiden a la región posicionarse efectivamente en la investigación y la economía mundial (OEA, 2005).

Para el BID Chile y México lideran en el continente el GCI (índice global de competitividad), en términos de competitividad, con datos de 2004 pero ocupan los puestos 32 y 48 respectivamente en relación con el resto del mundo (BID, 2005).

De igual manera, sólo Chile y Brasil superaron la media mundial para el año 2004 del NRI (índice de potencial para la conectividad) de penetración de las TIC para América Latina, pero ocuparon los puestos 32 y 39 respectivamente respecto al resto del mundo.

En este sentido, las prioridades para el desarrollo coherente de las redes de $\mathrm{I}+\mathrm{D}$ en América Latina deben enfocarse en las siguientes acciones estratégicas (OEA, 2005): a) Aumentar la inversión en redes avanzadas a fin de conectar todas las universidades y centros de investigación con una capacidad de, por lo menos, 100 Mpbs.

b) Promover marcos reguladores que fomenten infraestructura de redes avanzadas y de fácil acceso a nuevas tecnologías de investigación y educación.

c) Invertir en los procesos de investigación universitarios que permitan el fomento y desarrollo de redes.

d) Invertir en la formación de capital humano para la gestión y manejo de las redes avanzadas con el apoyo de empresas y asociaciones de telecomunicaciones.

e) Promover y difundir en las sociedades del continente la importancia de dichas redes para el desarrollo nacional y local.

f) Fortalecer y priorizar la ciberestructura de banda ancha en universidades, gobiernos y entes municipales.

En este escenario, las redes de I+D en América Latina tienden a unificarse tanto regional como internacionalmente como estrategia de expansión informativa y de conexión en la era del conocimiento, a pesar de la brecha cognitiva existente. Sin embargo, dicha brecha se relaciona con la desvinculación de los niveles de competitividad y productividad internacional que ponen a la ciencia y a la tecnología en un camino complejo de retos y transformaciones en las políticas de desarrollo del sector en el siglo que avanza. ${ }^{10}$

\section{LA UNIVERSIDAD COMO EJE DE LAS REDES DE I+D Y SU ROL EN LA INSERGIÓN DE ESTRATEGIAS HACIA EL USO DE LAS TIC EN AMÉRICA LATINA}

Es difícil destacar las configuraciones de las redes en América Latina a partir de su grado de amplitud debido a las múltiples conexiones electrónicas existentes en la actualidad; sin embargo, es posible generalizarlas desde el grado de configuración en los países donde se crean. ${ }^{11}$

10 Para profundizar en esta problemática y en dichas políticas, véase OEA (2005).

11 Se consideraron las redes avanzadas conectadas a la red CLARA hasta junio de 2006 a fin de facilitar las características esbozadas en este punto del trabajo, ya que posee la más importante conexión en la región. Puede verse la organización y amplitud de las mismas en la siguiente dirección electrónica: http://www.redclara.net/ 
La composición institucional de estas redes tiene en común una estrecha vinculación con las universidades públicas y pocos miembros de organismos nacionales y empresas privadas, por lo que la influencia de las primeras es muy marcada, tanto en el origen, desarrollo y participación como en la cooperación y producción de productos de $\mathrm{I}+\mathrm{D}$ en el continente.

En Argentina, de los 37 miembros de la Red Teleinformática Académica (RETINA), 12 son universidades. En Colombia, la mayoría de los miembros de la Red Nacional Académica de Tecnología Avanzada (RENATA) son universidades. En Chile, de las 16 instituciones con las que cuenta la Red Universitaria Nacional (REUNA), 14 son universidades. Es el mismo caso de la Corporación Universitaria para el Desarrollo de Internet (CUDI) en México, pues de sus 136 miembros, 54 son universidades. En Ecuador, de los 24 miembros del Consorcio Ecuatoriano para el Desarrollo de Internet Avanzado (CEDIA), 13 son universidades. En Perú, de los 7 miembros activos de la Red Académica Peruana (RAAP), 6 son universidades, al igual que en Venezuela, donde 34 de los 69 miembros de la REACCIUN (Red Académica de Centros de Investigación y Universidades) son de este ámbito.

Este dominio casi absoluto se aprecia también en Centroamérica. En Costa Rica, por ejemplo, de los 10 miembros relacionados con la Red Nacional de Investigación (CR 2 net), 4 son universidades. En Guatemala, 6 de los 7 miembros de la Red Avanzada Guatemalteca para la Investigación y Educación (RAGIE) provienen del campo universitario.

De igual modo, esta proporción se nota en países como El Salvador (Red Avanzada de Investigación, Ciencia y Educación Salvadoreña; RAICES), Nicaragua (Red Nicaragüense de Internet) y Honduras con la Universidad Tecnológica Latinoamericana, que concentra el eje en dicha acción.

El dominio de las universidades en estas redes proviene de las propias características de la educación superior en América Latina, que marchó aisladamente hasta la última década del siglo xx con los sistemas de ciencia y tecnología aunque, en la práctica, los investigadores universitarios trabajaban conjuntamente con otros organismos no universitarios en el proceso de desarrollo científico, pero sin una política coherente e integrada de esos esfuerzos de desarrollo del sector.

Por otro lado, estas redes se configuran a partir de áreas de acción caracterizadas por la promoción o difusión de actividades académicas, de investigación o de información en torno a vinculaciones de cooperación interinstitucional, regional e internacional (tabla 1).

Del mismo modo, las universidades dominan el espectro institucional de las redes pero son las que menos invierten en el sector de I+D; el caso contrario ocurre en los países desarrollados, donde la inversión proviene en su mayoría de la empresa privada, con muy bajos índices de gasto por parte de las instituciones universitarias.

En Estados Unidos, por ejemplo, en 2003, el 63\% de dicha inversión provino de sectores o fundaciones privadas, y sólo el 3,8\% de las instituciones de educación superior. En América Latina, en cambio, también en el mismo año, el sector gubernamental financió el 46,6\% de las actividades de $\mathrm{I}+\mathrm{D}$, en contraste con un $35,2 \%$ de la empresa privada y un $20,4 \%$ de las instituciones de educación superior (RICYT, 2005).

Sin embargo, dichas redes de $\mathrm{I}+\mathrm{D}$ contribuyen a la interconexión y a una mayor penetración de las TIC en la sociedad latinoamericana mediante la universidad como el centro de mayor intercambio social y tecnológico de la región.

En el continente, algunas universidades han llevado a la práctica estas ideas por medio de los centros de comunicación universitarios, que prestan apoyo en las comunidades en el uso de Internet a precios módicos e incluso, en ocasiones, gratuitos. Éstos son los casos de la Universidad Nacional de San Agustín y la Universidad Nacio- 
TABLA 1. Redes de I+D en América Latina y el Caribe por área de acción (año 2005)

\begin{tabular}{|c|c|c|}
\hline Área de acción & Red o centro de datos principal & País \\
\hline \multirow[t]{6}{*}{ Académica } & RAAP (Red Académica Peruana) & Perú \\
\hline & RAU (Red Académica Uruguaya) & Uruguay \\
\hline & REACCIUN (Red Académica de Centros de Investigación y Universidades) & Venezuela \\
\hline & RENATA (Red Nacional Académica de Tecnología Avanzada) & Colombia \\
\hline & RETINA (Red Teleinformática Académica) & Argentina \\
\hline & REUNA (Red Universitaria Nacional) & Chile \\
\hline \multirow[t]{4}{*}{ Sociedad de información Internet } & ADSIB (Agencia para el Desarrollo de la Sociedad de la Información en Bolivia) & Bolivia \\
\hline & CUDI (Corporación Universitaria para el Desarrollo de Internet) & México \\
\hline & CEDIA (Consorcio Ecuatoriano para el Desarrollo de Internet Avanzado) & Ecuador \\
\hline & Red Nicaragüense de Internet & Nicaragua \\
\hline & ATLANTEA & Puerto Rico \\
\hline & CALDAS & Colombia \\
\hline & CR 2 net (Red Nacional de Investigación) & Costa Rica \\
\hline & RAISES (Red Avanzada de Investigación, Ciencia y Educación Salvadoreña) & El Salvador \\
\hline & RNP (Red Nacional de Enseñanza e Investigación) & Brasil \\
\hline & RAGIE (Red Avanzada Guatemalteca para la Investigación y Educación) & Guatemala \\
\hline Educación superior (ministerial) & MES (Ministerio de Educación Superior) & Cuba \\
\hline $\begin{array}{l}\text { Educación superior } \\
\text { (portal institucional) }\end{array}$ & UNITEC (Universidad Tecnológica Latinoamericana) & Honduras \\
\hline Sistemas de ciencia y tecnología & ENACYT & Panamá \\
\hline
\end{tabular}

nal de San Antonio de Abad en Cuzco, en Perú; la Universidad Tecnológica de Panamá, la Universidad de la Frontera en Chile y la Universidad Metropolitana en México (Proenza et al., 2001).

Por otro lado, con el apoyo de centros de información públicos las universidades no solamente tienen una oportunidad de ofrecer logros en la reducción de la brecha digital; las redes de investigación, como vehículo de expansión del uso de las TIC en América Latina, deben enfocarse a partir del propio impacto que han generado éstas en la dinámica universitaria.

Estos impactos se orientan a los cambios del rol del docente universitario, al propio alumno, al proceso de enseñanza-aprendizaje, al currículo y a las propias institu- ciones (Torres, 2002; Salinas, 2004; Área, 2003, y Solbes et al., 2001).

No obstante, el impacto de las TIC en el rol docente ha sido tratado ampliamente por autores estudiosos en el campo educativo (Barberà et al., 2005; Romagnoli et al., 1999; Guzmán, 2004; Pariente, 2005, y Sánchez, 2003). Sin embargo, en estos estudios se ha notado una tendencia a no reflejar claramente el rol investigador del docente universitario en los análisis sobre el uso de las TIC en la educación superior.

Las razones parecen ser el desarrollo de la teoría de la instrucción y la tecnología educativa en los discursos explicativos sobre los roles docentes en la educación superior a partir de una perspectiva epistemológica funciona- 
lista, por un lado, y la poca integración entre la docencia y la investigación como consecuencia del currículo profesionalizante de las universidades latinoamericanas.

En la práctica universitaria actual, el rol de investigadordocente es una de las competencias y habilidades más importantes de la profesión universitaria (Martínez, 1999; Enao, 2002, y Callejas, 2002), y el punto de enlace de las redes de investigación de $\mathrm{I}+\mathrm{D}$ con el aula de clase. ${ }^{12}$

Cuando un docente está adscrito a una línea formal de investigación y enlazado en una red institucional, nacional o internacional de estudio, puede transferir dichas capacidades en sus actividades de cátedra integrando a sus alumnos en los proyectos concretos o asesorando tesis de pre y posgrado a partir de herramientas de información y comunicación.

En América Latina estas estrategias han ido desarrollándose en las redes ya existentes de $\mathrm{I}+\mathrm{D}$ en la formación y la alfabetización informática de los docentes universitarios. Tal es el caso de la red CUDI (Corporación Universitaria para el Desarrollo de Internet), en México, que desarrollará una red NREN dirigida a los docentes universitarios y de educación media. Otro proyecto referido a la formación de competencias en el manejo de las TIC en educación es la llamada Universidad Virtual, al igual que el Proyecto de Mejoramiento de la Calidad y Nivel de los Servicios Tecnológicos de Apoyo a la Docencia en las Universidades, auspiciados por la red REUMA (Red Universitaria Nacional) en Chile. En el caso de Brasil, la Red Nacional de Enseñanza e Investigación (RNP), conjuntamente con el Instituto de Matemática Pura Aplicada (IMPA), lanzó un programa de capacitación a distancia para maestros de secundaria.

De igual modo, en el continente ya se desarrollan algunas redes que operan en el ámbito de la educación básica y secundaria, y que tienen como propósito integrar a docentes, alumnos y comunidad en las TIC, con un fuerte apoyo técnico de las instituciones de educación superior.

Estas experiencias se desarrollan en países como Chile con la red Enlaces, en Argentina con la Red de Escuelas (RedEs), en Brasil con el Programa Nacional de Informática en la Educación (PROINFO), en México con la Red Escolar ${ }^{13}$ y en Venezuela con la Red Nacional de Actualización Docente Mediante la Informática y la Telemática (RENADIT).

En relación al rol del alumnado en esta vinculación con las redes de $\mathrm{I}+\mathrm{D}$, es propia de la mayoría de las universidades latinoamericanas la poca flexibilidad del currículo en torno al perfil de los egresados del sistema de educación superior. Los alumnos, en la mayoría de las carreras, no desarrollan competencias ni habilidades en el campo de la investigación desde que inician la vida universitaria, salvo en las asignaturas obligatorias de metodología del estudio y el trabajo de licenciatura, por lo que la desvinculación entre la actitud investigadora y el rol del alumnado son inminentes en los ejes curriculares.

Ungerfeld (2003) afirma que la investigación enfrenta al alumno con nuevas situaciones, con lo que aumenta su acción y comprensión, y se estimula el desarrollo de personalidades más creativas, la autoconfianza y la motivación. De igual modo, incentiva la cultura de la indagación de fuentes electrónicas, y descubre aprendizajes distintos a los libros y las prácticas repetitivas de las asignaturas cuando se acompaña con una herramienta TIC.

En dos casos regionales, específicamente en Argentina y Venezuela, esta desvinculación es clara en la mayoría de las universidades, ya que se presenta un poco uso efectivo de las TIC tanto en la producción de conocimiento sobre el área como en la formación del profesorado en

\footnotetext{
12 Sin embargo, la problemática de las actitudes y el papel de la investigación en las universidades de América Latina constituye un obstáculo que debe vencerse y un punto de reflexión posterior que tiene que considerarse.

${ }_{13}$ Para un mayor conocimiento de estos proyectos, consúltese: http://www.esc.edu.ar/redes/estudio_espanol.htm
} 
el uso de estas herramientas en investigación. Del mismo modo, en el país austral, se evidencia una poca integración de las universidades con las redes científicas a partir de este tipo de herramientas (Finquelievich et al., 2005).

Esta desunión es todavía mayor en las universidades venezolanas dada «la desconfianza que aún existe en relación con la educación virtual, la poca cultura informática en la comunidad universitaria y, en general, la escasa disponibilidad de centros para el acceso a las TIC» (Cursi, 2003, pág. 57).

Parece claro que, para aprovechar las redes de $\mathrm{I}+\mathrm{D}$ en la formación de competencias sobre la investigación tanto en docentes como en alumnos, es necesario integrar en el currículo esta estrategia mediante posibles cluster de investigación por ejes de trabajo interdisciplinario. Esto debe llevarse a cabo ya desde el inicio de la carrera universitaria y ajustando al diseño instruccional herramientas de tecnologías de la información y de comunicación orientadas al estímulo de la creatividad (Borroto, 2004), al desarrollo cognitivo (Tascón, 2004) y a la instrucción constructiva de cuarta generación, como estrategia de culturización tecnológica de los docentes, los alumnos, las propias instituciones de educación superior y la sociedad en general.

No obstante, estas experiencias en educación superior están iniciándose lentamente en muy pocos países de la región debido a que es necesario contar con una red avanzada de I+D que la mayoría de las naciones no posee, por lo que se espera un escenario poco optimista en torno a la masificación de esta estrategia para el desarrollo de competencias docentes en el manejo de las TIC en este ámbito, por lo menos en la primera década del presente siglo.

\section{CONCLUSIONES}

Los impactos más significativos de la sociedad de la información en el mundo globalizado de hoy se dirigen hacia la educación superior como mecanismo de transferencia efectiva de los valores inherentes a la información y comunicación para el advenimiento del desarrollo social. Esta importancia ha hecho que, desde una visión mundial, se la considere un bien de intercambio económico social que ha generado expectativas y críticas en los sectores más conservadores de la sociedad.

A pesar de esas posturas, se afianza el consenso mundial al considerar las TIC como sistemas válidos de desarrollo y progreso humano no sólo en el campo educativo, sino en todas las esferas de producción económica y social. Sin embargo, su uso y penetración en la actualidad operan en un contexto desigual generador de una brecha digital que impide el óptimo afianzamiento de las mismas en la mejora de la calidad de vida de los sectores más pobres y excluidos.

En este contexto, el nacimiento de la sociedad en red definió nuevas vinculaciones de intercambio científico tecnológico en el ámbito mundial y continental, y formalmente abrió las puertas a las redes electrónicas de $\mathrm{I}+\mathrm{D}$.

En América Latina, la red CLARA constituye el mecanismo regional hacia la interconexión con todas las redes avanzadas de $\mathrm{I}+\mathrm{D}$ del mundo y repercute en el aumento gradual de la cooperación regional e internacional dentro de un contexto globalizador. Dicha integración provino de los cambios en los sistemas de ciencia y tecnología a finales de los años noventa, cuando la unificación entre los organismos públicos y privados para el desarrollo de políticas integrales de producción tecnológica incidieron en los procesos de innovación, organización, cooperación y desarrollo de estrategias TIC en el continente.

Sin embargo, dichas redes aún no se han afianzado en la totalidad de la región y han dejado pocas experiencias positivas en el diseño, implantación, gestión y mantenimiento de las mismas. Países como Brasil, conjuntamente con Argentina, México y Chile, conforman un eje de 
desarrollo muy destacado no sólo en lo relativo a la productividad de dichas redes, sino a su ampliación, y al uso y penetración de las TIC en la sociedad en general.

Esta brecha cognitiva entre los países de la región en torno a la producción tecnológica y de cooperación internacional indica que a mayor solidez de una red de I+D avanzada, mayor es la producción de productos tecnológicos y de innovación en la nación que las tenga. Esto, por supuesto, está relacionado con otros factores, como la coherencia en las políticas que la fomenten y el financiamiento necesario que la mantenga.

Según los resultados esbozados, las redes de $\mathrm{I}+\mathrm{D}$ son una oportunidad para el uso y penetración de las TIC en las sociedades de América Latina. Las experiencias encontradas apuntan al poco uso de éstas en la vinculación investigación-docencia, en la apropiación tecnocultural de las TIC tanto en el entorno interno (comunidad universitaria) como en el externo (comunidad en general), y en el desarrollo de la competitividad de los países de la región en la economía mundial.

En este ámbito, se cree pertinente profundizar en los impactos de las redes de $\mathrm{I}+\mathrm{D}$ en los índices de productividad científica, en los gastos y en la inversión en su proporción con el PIB, en los niveles de cooperación regional e internacional, y en su papel en el fortalecimiento de la competitividad de los sistemas nacionales de ciencia y tecnología, así como en el apoyo de dichas redes a la reducción de la brecha digital y la lucha contra la pobreza en América Latina.

\section{BIBLIOGRAFÍA}

ABELLO, Raimundo; PÁEZ Javier; DACUNHA, Claudia (2001). "¿Son la ciencia y la tecnología un instrumento de desarrollo? Un análisis de caso para América Latina». Investigación y Desarrollo. Vol. 9, n. 1, pág. 372-387.

ÁREA, Manuel (2003). «De los web educativos al material didáctico web». Comunicación y Pedagogía. ‥ ${ }^{\circ}$ 188, pág. 32-38.

BANCO INTERAMERICANO DE DESARROLLO (2003). Construir sociedades del conocimiento: Nuevos desafíos de la educación terciaria [documento en línea]. BID. [Fecha de Consulta: 01/02/2006]. <http://siteresources.worldbank.org/EDUCATION/Resources/2782001099079877269/5476641099079956815/CKS-spanish.pdf>

BANCO INTERAMERICANO DE DESARROLLO (2005). Difusión de las tecnologías de información y comunicación para el desarrollo: Un marco para la acción [documento en línea]. BID. [Fecha de consulta: 01/06/2006]. <http://www.iadb.org/sds/doc/PerfilEstrategiadeTICparaeldesarrollo.pdf>

BARBERÀ, Elena; BADIA, Antoni (2005). «El uso educativo de las aulas virtuales emergentes en la educación superior» [artículo en línea]. Revista de Universidad y Sociedad del Conocimiento (RUSC). Vol. 2, no 2. UOC. [Fecha de consulta: 20/01/06]. <http://www.uoc.edu/rusc/2/2/dt/esp/barbera.pdf >

BORROTO, Gerardo (2004). «Las tecnologías de la información y las comunicaciones en la educación de la creatividad». Contexto Educativo [artículo en línea]. N. ${ }^{\circ}$ 31, año V. REIE. [Fecha de consulta: 23/01/06].

$<$ http://contexto-educativo.com.ar/2004/2/nota-05.htm> 
CALLEJAS, María (2002). "La investigación en la formación del profesor universitario: entre la teoría y la práctica». Colombia Ciencia y Tecnología. Año 20, n. 4, pág. 3-12.

CECCHINI, Simone (2005). Oportunidades digitales, equidad y pobreza en América Latina: ¿qué podemos aprender de la evidencia empírica? [documento en línea]. ONU. [Fecha de consulta: 10/06/2006].

<http://www.eclac.cl/publicaciones/Estadisticas/9/LCL2459PE/lc12459e.pdf>

COMISIÓN ECONÓMICA SOCIAL PARA AMÉRICA LATINA (CEPAL) (2005). Las nuevas tecnologías de la información y de la comunicación (TIC) y la institucionalidad social. Hacia una gestión basada en el conocimiento. María Rebeca Yánez; Pablo Villatoro (comps.) [documento en línea]. CEPAL. [Fecha de consulta: 12/06/2006]. <http://www.eclac.cl/publicaciones/DesarrolloSocial/8/LCL2298PE/sps_108.pdf>

CURSI, Renata (2003). Diagnóstico de la educación virtual en Venezuela [documento en línea]. Caracas: Universidad Metropolitana/IESAL, UM. [Fecha de consulta 12/01/2006].

<http://www.iesalc.unesco.org.ve/programas/internac/univ_virtuales/venezuela/vir_ve.pdf>

DOMÍNGUEZ, Mario (2003). «Las tecnologías de la información y la comunicación: sus opciones, sus limitaciones y sus efectos en la enseñanza». Nómadas [artículo en línea]. N. ${ }^{8}$. [Fecha de consulta: 10/01/2006].

$<$ http://www.ucm.es/info/nomadas/>

ENAO, Miriam (2002). «El papel de la investigación en la formación universitaria». Colombia Ciencia y Tecnología. Año 20, n. ${ }^{\circ}$, pág. 13-18.

FINQUELIEVICH, Susana; PRINCE, Alejandro (2005). Las universidades argentinas en la sociedad del conocimiento [documento en línea]. [Fecha de consulta: 10/11/2005].

<http://www.links.org.ar/infoteca/universidad-tics.pdf>

GUZMÁN, María (2004). «Estudio sobre los usos didácticos, procesos formativos y actitudes de los docentes universitarios en relación a Internet». Revista Iberoamericana de Educación [artículo en línea]. OEI. [Fecha de consulta: $09 / 01 / 2006]$.

<http://www.campus-oei.org/revista/deloslectores/633Guzman.pdf>

HERNÁNDEZ, Julieta; VÁSQUEZ, Jaime (2004). «Experiencia de la utilización de TIC en procesos de formación pedagógica de tutores». En: Primer Congreso Virtual Latinoamericano de Educación a distancia [página web en línea]. Latin Educa2004. [Fecha de consulta: 25/10/2005].

<http://www.latineduca2004.com/latineduca/arg/>

MARTÍNEZ, Felipe (1999). «¿Es posible una formación sistemática para la investigación educativa? Algunas reflexiones». Revista Electrónica de Investigación Educativa [artículo en línea]. Vol. 1, n. ${ }^{\circ}$ 1. REIE. [Fecha de consulta: 24/11/2005].

<http://redie.ens.uabc.mx/vol1no1/contenido-mtzrizo.html> 
OLAZARÁN, Mikel; TORRES, Cristóbal (1999). «Modelos del cambio científico: Una propuesta integradora». Nómadas [documento en línea]. N. ${ }^{\circ} 0$. [Fecha de consulta: 10/11/2005].

<http://www.ucm.es/info/nomadas/>

ORGANIZACIÓN DE LOS ESTADOS AMERICANOS (2005). Ciencia, tecnología, ingeniería e innovación para el desarrollo: Una visión para las Américas en el siglo XXI [documento en línea]. OEA. [Fecha de consulta: 11/06/2006]. $<$ http://www.oest.oas.org/engineering/espanol/documentos/esp_web_ok.pdf>

ORGANIZACIÓN DE ESTADOS AMERICANOS (2006). Declaración de Santo Domingo: Gobernabilidad y desarrollo en la sociedad del conocimiento [página web en línea]. OEA. [Fecha de consulta: 09/06/2006].

$<$ http://www.oas.org/main/main.asp?sLang=S\&sLink=http://www.oas.org/36ag>

ORGANIZACIÓN DE LAS NACIONES UNIDAS PARA LA EDUCACIÓN, LA CIENCIA Y LA CULTURA. UNESCO (2004). Educación superior en una sociedad mundializada [documento en línea]. Unesco. [Fecha de consulta: $03 / 02 / 2006]$.

$<$ http://www.unesco.org.ve/pruebaobservatorio/documentos $\% 20$ pdf/educaci\%F3n $\% 20$ superior $\% 20$ en $\% 20$ una $\% 20$ sociedad $\% 20$ mundializada.pdf>

ORGANIZACIÓN DE LAS NACIONES UNIDAS PARA LA EDUCACIÓN, LA GIENCIA Y LA CULTURA. UNESCO (2005). Hacia las sociedades del conocimiento [documento en línea]. Unesco. [Fecha de consulta: 03/06/2006]. <http://unesdoc.unesco.org/images/0014/001419/141908s.pdf>

PARIENTE, Francisco (2005). «Hacia una auténtica integración curricular de las tecnologías de la información y comunicación». Revista Iberoamericana de Educación [artículo en línea]. OEI. [Fecha de consulta: 09/01/2006]. $<$ http://www.campus-oei.org/revista/deloslectores/1055Pariente.pdf>

PROENZA, Francisco; BASTIDAS, Roberto; MONTERO, Guillermo (2001). Telecentros para el desarrollo socioeconómico y rural en América Latina y el Caribe [documento en línea]. BID. [Fecha de consulta: 04/03/2006].

$<$ http://www.e-paratodos.org/pdf/Telecentros.pdf>

RED DE INDICADORES DE CIENCIA Y TECNOLOGÍA-IBEROAMERICANA E INTERAMERICANA (RICYT) (2005). Estadísticas centrales de ciencia y tecnología [página web en línea]. RICYT. [Fecha de consulta: 15/10/2005]. $<$ http://www.ricyt.edu.ar/>

ROMAGNOLI, Claudia; FEMENIAS, Guillermo; CONTE, Paola (1999). Internet, un nuevo recurso para la educación. Chile: Ministerio de Educación.

SALINAS, Jesús (2004). «Innovación docente y uso de las TIC en la enseñanza universitaria». Revista de Universidad y Sociedad del Conocimiento (RUSC). [artículo en línea]. Vol. 1, nº 1. UOC. [Fecha de consulta: 27/01/2006]. $<$ http://www.uoc.edu/rusc/dt/esp/salinas1104.pdf $>$ 
SÁNCHEZ, Germán (2004). «Los sistemas de ciencia y tecnología en tensión: Su integración al patrón de reproducción global». Convergencia [artículo en línea]. Vol. 11, n. 35, pág. 193-220. [Fecha de consulta: 10/12/2005]. <http://www.uaemex.mx/webvirtual/wwwconver/htdocs/rev35/35pdf/7_GERMAN_SANCHEZ.pdf>

SÁNCHEZ, Jaime (2003). Usos educativos en Internet. Universidad de Chile [documento en línea]. Universidad de Chile. [Fecha de consulta: 12/12/2005].

<http://www.dcc.uchile.cl/ jsanchez/Pages/papers/usoseducativosdeinternet.pdf>

SEBASTIÁN, Jesús (1999). «Las redes de cooperación como modelo organizativo y funcional para la I+D». Redes. Vol. 7, n. ${ }^{\circ}$ 15, pág. 97-111.

SOLBES, Jordi; VILCHES, Amparo; GIL, Daniel (2001). El enfoque CTS en la formación del profesorado. En: Pedro Membiela (ed.). Enseñanza de las ciencias desde la perspectiva ciencia-tecnología y sociedad. Madrid: NARCEA. Pág. 163175.

TASCÓN, Claudio (2004). «La potenciación de aprendizajes en un entorno TIC: los mapas conceptuales como instrumento cognitivo y herramienta de aprendizaje visual». En: Alberto J. Cañas; Joseph D. Novak; Fermín M. González (eds.). Concept Maps: Theory, Methodology, Technology Proc. of the First Int. Conference on Concept Mapping. Pamplona.

TORRES, Cristóbal (2002). «El impacto de las nuevas tecnologías en la educación superior: un enfoque sociológico». Boletín de la Red Estatal de Docencia Universitaria [documento en línea]. Vol. 2, n. ${ }^{\circ}$ 3. UIT. [Fecha de consulta: 10/01/2006].

<http://www.uc3m.es/uc3m/revista/VOL2NUM3/Indice.htm>

UNGERFELD, Rodolfo (2003). «La investigación como soporte de actividades de enseñanza universitaria». Contexto Educativo [artículo en línea]. Año V, n. ${ }^{\circ} 31$. REIE. [Fecha de consulta: 23/01/2006].

$<$ http://contexto-educativo.com.ar/2004/2/nota-04.htm>

UNIÓN INTERNACIONAL DE TELECOMUNICACIONES (2003). Community access indicators. Extract adapted from chapter 2 of the 2003 World Telecommunication Development Report. Access indicators for the information society on «Measuring access to ICTs» [documento en línea]. UIT. [Fecha de consulta: 04/02/2006].

<http://www.itu.int/ITU-D/ict/mexico04/doc/index-es.html>

UNIÓN INTERNACIONAL DE TELECOMUNICACIONES (2006). Informe sobre el desarrollo mundial de las telecomunicaciones-TIC. Evaluación de las TIC para el desarrollo económico y social [documento en línea]. UIT. [Fecha de consulta: 02/06/2006].

$<$ http://www.itu.int/itunews / manager/display.asp?lang=es\&year=2006\&issue=02\&ipage=big_picture\&ext=html $>$ 
Para citar este documento, puedes utilizar la siguiente referencia:

ROYERO, Jaim D. (2007). «Las redes de I+D como estrategia de uso de las TIC en las universidades de América Latina» Revista de Universidad y Sociedad del Conocimiento (RUSC) [artículo en línea]. Vol. 3, n. ${ }^{\circ}$ 2. UOC. [Fecha de consulta: $\mathrm{dd} / \mathrm{mm} / \mathrm{aa}]$.

<http://www.uoc.edu/rusc/3/2/dt/esp/royero.pdf >

ISSN 1698-580X

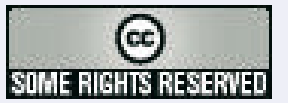

Los textos publicados en esta revista están sujetos -si no se indica lo contrario- a una licencia de Reconocimiento 3.0 España de Creative Commons. Puede copiarlos, distribuirlos, comunicarlos públicamente y hacer obras derivadas siempre que reconozca los créditos de las obras (autoría, nombre de la revista, institución editora) de la manera especificada por los autores o por la revista. La licencia completa se puede consultar en http://creativecommons.org/licenses/by/3.0/es/deed.es.

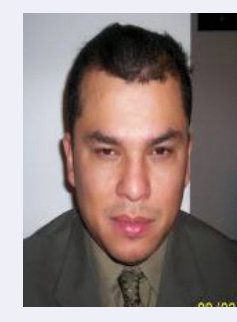

\section{Jaim D. Royero Rivera}

Docente de posgrado de la Universidad Gran Mariscal de Ayacucho (Venezuela)

rojada@cantv.net

Licenciado en Educación, Mención Planificación Educativa por la Universidad Central de Venezuela (UCV), máster en Ciencias de la Educación Superior por la Universidad de la Habana-CEPES y candidato al doctorado en Educación.

Docente y coordinador de las tutorías de grado del posgrado en Gerencia Educativa de la Universidad Gran Mariscal de Ayacucho (Venezuela).

Asesor educativo y experto en gestión de sistemas de investigación y vinculación tecnológica en sistemas de educación superior.

Jaim D. Royero ha dictado cursos de gestión de la investigación y otras áreas de la gerencia universitaria. Ha publicado artículos en la Revista Iberoamericana de Educación y otros trabajos de interés en la Web.

Investigador a tiempo completo en el IUTJAA (Instituto Universitario de Tecnología José Antonio Anzoátegui), en Venezuela. Autor del proyecto Sistema Institucional de Investigación (SIDI) de esta institución y asesor permanente de su consejo. 